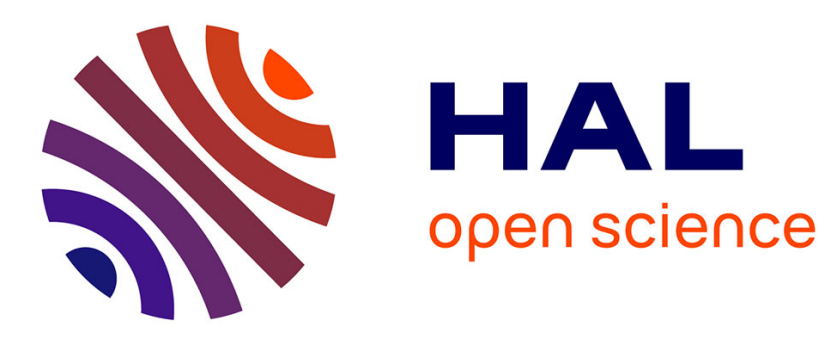

\title{
A Digital-Controller Parameter-Tuning Approach, Application to a Switch-Mode Power Supply
}

Xuefang Lin-Shi, Florent Morel, Bruno Allard, Dominique Tournier, Jean-Marie Rétif, Shuibao Guo, Yanxia Gao

\section{To cite this version:}

Xuefang Lin-Shi, Florent Morel, Bruno Allard, Dominique Tournier, Jean-Marie Rétif, et al.. A Digital-Controller Parameter-Tuning Approach, Application to a Switch-Mode Power Supply. IEEE ISIE, Jun 2007, Vigo, Spain. pp.3356 - 3361, 10.1109/ISIE.2007.4375154 hal-00369454

\section{HAL Id: hal-00369454 https://hal.science/hal-00369454}

Submitted on 3 Jun 2009

HAL is a multi-disciplinary open access archive for the deposit and dissemination of scientific research documents, whether they are published or not. The documents may come from teaching and research institutions in France or abroad, or from public or private research centers.
L'archive ouverte pluridisciplinaire HAL, est destinée au dépôt et à la diffusion de documents scientifiques de niveau recherche, publiés ou non, émanant des établissements d'enseignement et de recherche français ou étrangers, des laboratoires publics ou privés. 


\title{
A Digital-Controller Parameter-Tuning Approach, Application to a Switch-Mode Power Supply
}

\author{
Xuefang Lin-Shi, Florent Morel, Student Member, IEEE, Bruno Allard, Senior Member, IEEE, \\ Dominique Tournier, Jean-Marie Rétif \\ AMPERE, CNRS UMR 5005, INSA-Lyon \\ Building L. De Vinci, 20 avenue A. Einstein, F-69621 Villeurbanne Cedex, France \\ Email: $\{$ xuefang.shi, florent.morel, bruno.allard, dominique.tournier, jean-marie.retif $\} @$ insa-lyon.fr \\ Shuibao Guo, Yanxia Gao \\ Institute of Electrical and Control Engineering, Shanghai University \\ 149 Yanchang Road, 200072 Shanghai, China \\ Email: shuibao.guo@insa-lyon.fr, eegoanna@163.com
}

\begin{abstract}
Analogue control of monolithic DC/DC converters is technologically coming to a limit due to high switching frequency and a request for large regulation bandwidth. Digital control is now experimented for low-power low-voltage switch-mode power supply. Digital implementation of analogue solutions does not prove real performances. This paper compares a classical digital controller to a candidate alternative strategy. Sensitivity functions are used to compare controller performances. An off-line approach using fuzzy logic to quantify controller performances and a genetic algorithm to obtain an optimal controller is presented. A so-called RST algorithm optimized with this approach shows better performances.
\end{abstract}

\section{INTRODUCTION}

For many years now, there is a trend to embed power management unit inside portable devices like cellphone, personal digital assistant or MP3-player. Most portable devices use a battery of voltage between $5.5 \mathrm{~V}$ when in charge, $3.3 \mathrm{~V}$ during discharge lifetime and down to $2.7 \mathrm{~V}$ when empty. Devices embed various functions supplied from various voltages. Processors require $1.8 \mathrm{~V}$ down to $1.2 \mathrm{~V}$ while backlight led system require $20 \mathrm{~V}$ at least. Non isolated DC/DC converters are considered in place of low-drop out regulators for the sake of efficiency. This paper will now address only stepdown conversion and associated buck architecture or stepdown switch-mode power supply (SMPS).

An example of full-analogue synchronous buck converter is pictured in Fig. 1. Except the passive L-C output filter, all blocks are integrated monolithically using CMOS standard technology [1], [2]. A 2-pole/2-zero compensator is implemented to achieve a maximal regulation bandwidth, maximal transient performance and maximal accuracy.

A $100 \mathrm{MHz}$ SMPS, $80 \%$ peak efficiency, $20 \mathrm{MHz}$ regulation bandwidth is presented in [3]. The design is compatible with standard CMOS process. Whatever the analogue control presents some limitations. First of all, the design of the compensator is not automated and the design engineer needs to take care of a trade-off between performances, accuracy and stability [4]. When R-C constant have been set, manufacturing introduces deviation with respect to design values and calibrations are required. The robustness is not sufficient, so a lot of

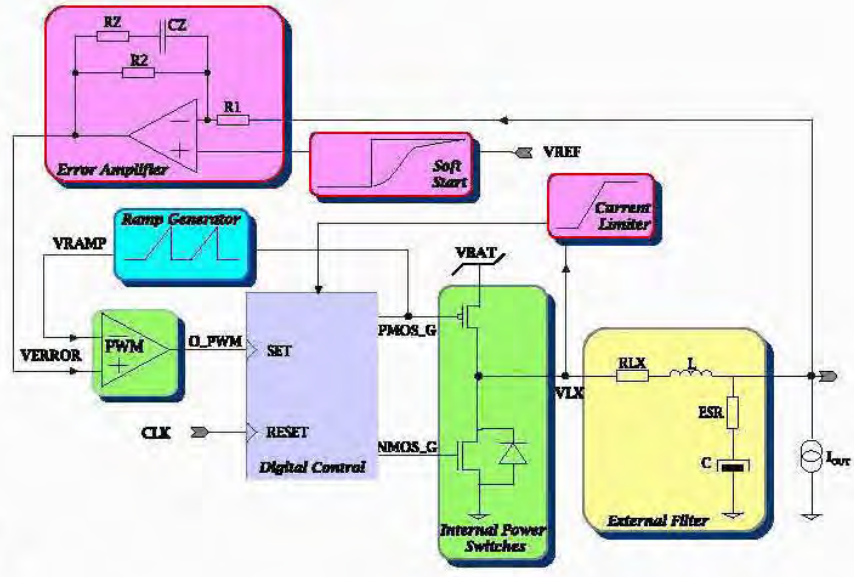

Fig. 1. Schematic synchronous step-down SMPS

efforts are put on alternative approaches as digital control.

Digital control is not new in the field of Power Electronics. It is often associated with DSP or other processorlike implementation [5]-[8]. Generally the digital control system presents sufficient resources to accommodate the modest switching frequency of the converter, in the $\mathrm{kHz}$ range. In embedded applications, switching frequencies in the $\mathrm{MHz}$ and plus range are necessary in order to reduce the size of passive components [9].

Due to the cost/complexity constrains existing in smallpower dc-dc converters with integrated digital controller, most published papers consider a discrete-time scheme equivalent to the analogue compensator (such as PID controller) [10], [11].

In order to satisfy the constrains on the load variation to achieve high transient performance and accuracy, an autotuning process should be introduced. Some publications about auto-tuning of digital PID controller for DC/DC converters can be found in [12]-[14]. However, most of the solutions are on-line tuning, so they require an increase of the silicon area of the IC controller. The practical use in very high frequency 


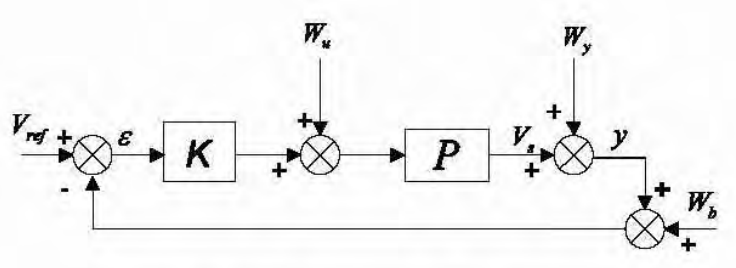

Fig. 2. Block diagram of the SMPS

$(>500 \mathrm{kHz})$ and very low power $(<1 \mathrm{~W})$ is still questionable. In this paper, an off-line tuning RST controller is presented. With similar complexity as a classical PID controller, a socalled RST controller presents more degrees of freedom for improving nominal and robust performances and rejection of disturbances [15]. Based on pole placement combined with the shaping of sensitivity functions, the paper investigates an off-line automated approach by using fuzzy logic and genetic algorithm to correctly specify the desired performances by adjusting the sensitivity functions in the frequency domain where it is necessary. As the automated parameter determination is performed off-line, there is no need supplementary silicon area for the auto-tuning scheme.

The paper is organized as follows: Section II reviews sensitivity functions and the application of the sensitivity function analysis on a buck converter. Section III introduces a digital PID controller for comparison purpose. The robust RST digital controller and the off-line automated tuning approach are described in Section IV. Simulation results are detailed in Section V.

\section{Sensitivity Functions}

In order to quantify system dynamics, robustness and noise rejection properties of tested controllers, sensitivity functions are introduced.

Fig. 2 represents the model of a SMPS $(P)$ and its controller $(K)$ when adding a control noise $W_{u}$ (e.g. PWM noise), an output noise $W_{y}$ (e.g. load variations) and a measurement noise $W_{b}$ (e.g. A/D converter noise).

From Fig. 2, it comes the following relation that leads to the sensitivity functions.

$$
\begin{aligned}
y & =\Gamma \cdot V_{r e f}+S_{y y} \cdot W_{y}+S_{y b} \cdot W_{b}+S_{y u} \cdot W_{u} \\
\Gamma & =\frac{K P}{1+K P}=\frac{L_{y y}}{1+L_{y y}} \\
S_{y y} & =\frac{1}{1+K P}=\frac{1}{1+L_{y y}} \\
S_{y b} & =\frac{-K P}{1+K P}=\frac{-L_{y y}}{1+L_{y y}} \\
S_{y u} & =\frac{P}{1+K P}=\frac{P}{1+L_{y y}}
\end{aligned}
$$

where $\Gamma$ is the closed loop transfer function, $S_{y y}, S_{y b}$ and $S_{y u}$ are respectively the output-to-output, measure-to-output and control-to-output sensitivity functions.

Constraints or disturbance rejections are naturally expressed in terms of frequency sensitivity shapes. For a given controller,

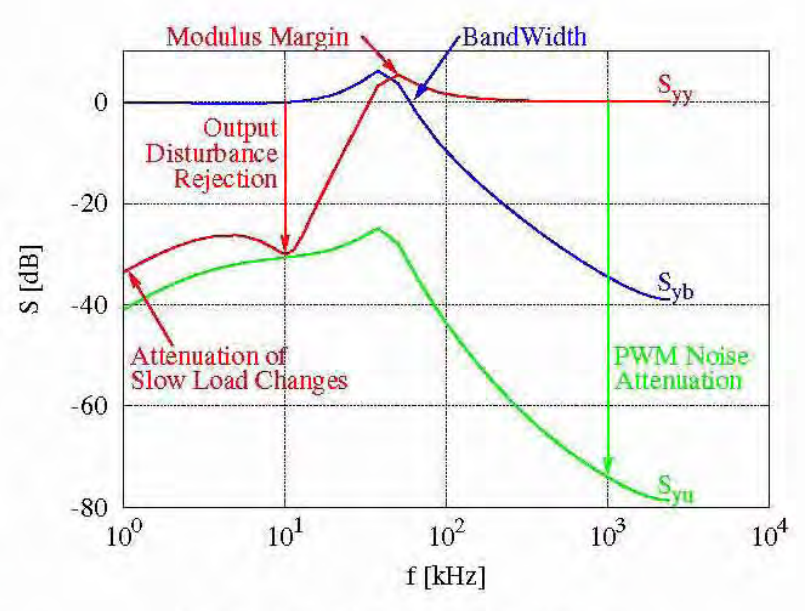

Fig. 3. Example of Sensitivity Functions

the sensitivity functions allow to evaluate the controller behavior in relation to the desired attenuation constraints.

Fig. 3 is an example of the gain plot of sensitivity functions. PWM and output noise attenuations are pointed considering a $1 \mathrm{MHz}$ PWM frequency and a $1 \mathrm{kHz}$ output disturbance resonance. The gradient of $S_{y y}$ at low frequency determines the dynamic behavior of the system. The bandwidth of $S_{y b}$ defines the influence of measurement noise on the output voltage and the closed loop bandwidth since it has the same transfer function as $\Gamma$ expect for the sign. The gain of $S_{y z}$ verifies the rejection of control perturbations such as the PWM-related noises.

In addition, from the maximum value of $S_{y y}$, the modulus margin can be determined. Indeed, it can be shown that the maximum value of $S_{y y}$ is proportional to the inverse of the modulus margin [16]. The modulus margin $\Delta \mathrm{M}$ is defined as the minimum distance of $L_{y y}$ with respect to the critical locus (-1) in the Nyquist plan. The modulus margin and delay margin quantify the robustness of the modeling uncertainties. The delay margin $\Delta \tau$ is deduced from the phase margin $\Delta \Phi$ by $\Delta \tau=\frac{\Delta \Phi}{\omega_{\Phi}}$, where $\omega_{\Phi}$ is the pulsation of phase margin determination.

In order to ensure robustness, the modulus margin $\Delta \mathrm{M}$ is kept higher than 0.5 and the delay margin must be higher than the sampling period (to ensure that the delay induced by controller computing time does not lead to unstable operation).

\section{PID CONTROL}

The PID controller is presented for comparison purpose with the RST controller. A digitally controlled buck converter operating in continuous conduction mode (CCM) can be regarded as a second order discrete-time system [17]:

$$
P(z)=\frac{b_{1} z+b_{2}}{z^{2}+a_{1} z+a_{2}}
$$

A discrete-time PID controller can be written as:

$$
K_{P I D}(z)=\frac{r_{0} z^{2}+r_{1} z+r_{2}}{(z-1)\left(z+s_{1}\right)}
$$




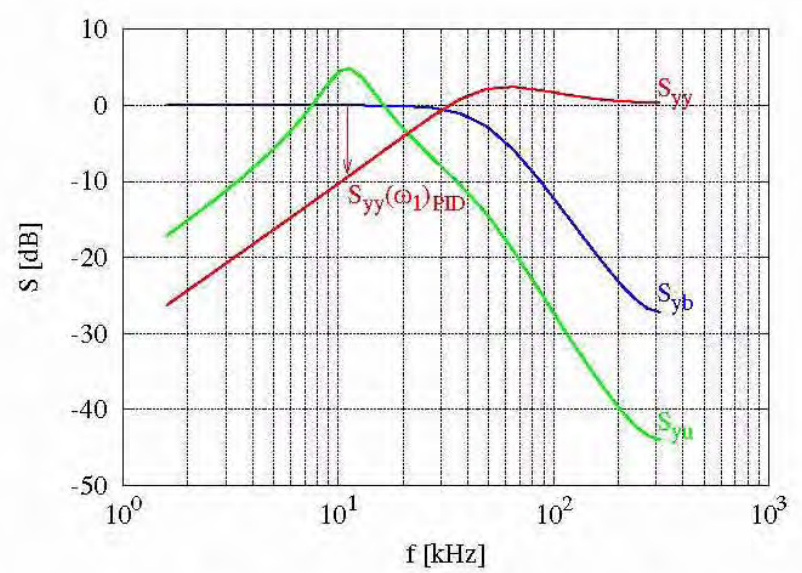

Fig. 4. Sensitivity functions for the PID controlled system

where $r_{0}, r_{1}, r_{2}, s_{1}$ are the controller parameters to be determined.

By cancelling the poles of $P(z)$ with the zeros of $K(z)$, the closed-loop reference for the output voltage transfer function is:

$$
\begin{aligned}
\Gamma & =\frac{K P}{1+K P} \\
& =\frac{r_{0} b_{1} z+r_{0} b_{2}}{z^{2}+\left(s_{1}-1+r_{0} b_{1}\right) z+\left(r_{0} b_{2}-s_{1}\right)} \\
& =\frac{r_{0} b_{1} z+r_{0} b_{2}}{z^{2}+p_{1} z+p_{2}}
\end{aligned}
$$

where $p_{1}$ and $p_{2}$ are defined by the desired closed-loop dynamics which correspond to second order dynamics with a pulsation $\omega_{c l}$ and a damping ratio $\xi_{c l}$. The conditions for the cancellation of the poles of $P(z)$ by the zeros of $K(z)$ are:

$$
\begin{aligned}
& r_{0}=\frac{1+p_{1}+p_{2}}{b_{1}+b_{2}} \\
& r_{1}=a_{1} r_{0} \\
& r_{2}=a_{2} r_{0} \\
& s_{1}=r_{0} b_{2}-p_{2}
\end{aligned}
$$

The circuit elements are $\mathrm{L}=10 \mu \mathrm{H}, \mathrm{C}=22 \mu \mathrm{F}, \mathrm{R}=$ $3 \Omega, \mathrm{V}_{B A T}=3 \mathrm{~V}$ and the sampling frequency is set to $\mathrm{f}_{e}=625 \mathrm{kHz}$. The desired closed-loop pulsation must be smaller than the Nyquist frequency, so it is set to $311850 \mathrm{rd} / \mathrm{s}$ corresponding to 4.5 times the open-loop pulsation. With the closed-loop damping ratio of 0.7 , the controller parameters are $r_{0}=5.23, r_{1}=-10.1, r_{2}=4.93$ and $s_{1}=-0.471$. The corresponding sensitivity functions and Nyquist plot of $L_{y y}$ are presented respectively in Fig. 4 and Fig. 5. For an output disturbance with a pulsation of $\omega_{1}$ (LC filter resonance), the gain of $S_{y y}$ on $\omega_{1}$ gives the information on the disturbance rejection. In the studied case, $\omega_{1}=11 \mathrm{kHz}$. It can be seen that $S_{y y}\left(\omega_{1}\right)$ is about $-8 \mathrm{~dB}$. Concerning the stability robustness, the modulus, phase and delay margins are $\Delta \mathrm{M}_{P I D}=0.75$, $\Delta \Phi_{P I D}=64^{\circ}, \Delta \tau_{P I D}=3.63 \mathrm{~T}_{e}$. Due to the sampling effect,

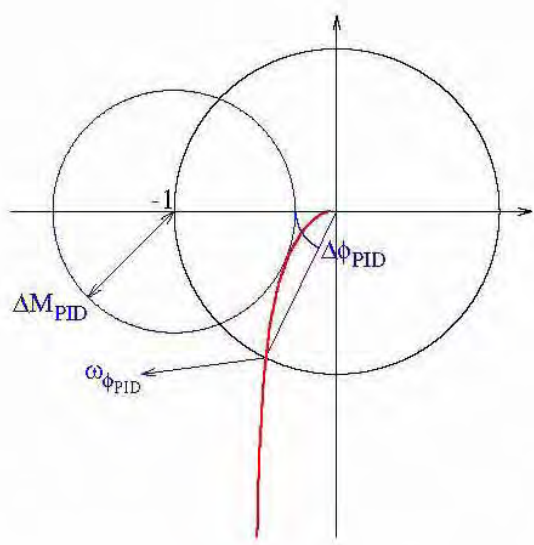

Fig. 5. Nyquist plot of $L_{y y}$ for the PID controlled system

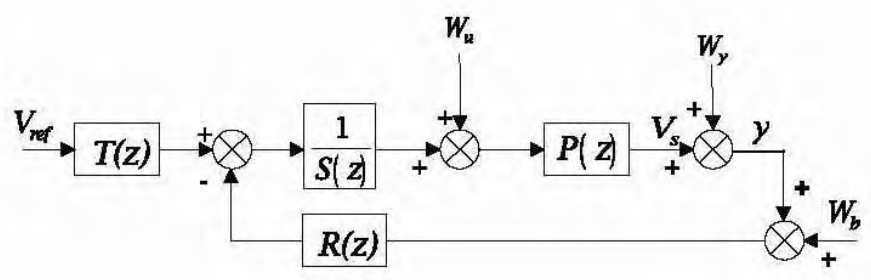

Fig. 6. RST control structure

sensitivity functions are plotted until the Nyquist frequency. As the PWM frequency is set equal to the sampling frequency $f_{e}$, the PWM noise rejection can not be appreciated. The overall tuning of the PID controller is quite classical.

\section{ROBUST RST CONTROL}

RST control realizes a relevant approach for linear Single Input Single Output (SISO) systems [15]. A RST controller is considered here in order to obtain a better output disturbance rejection while keeping a good PWM noise rejection and a good robustness. The structure of a RST control is presented in Fig. 6.

If the discrete time SMPS model is described by the transfer function $P(z)=\frac{B(z)}{A(z)}$, where $B(z)$ and $A(z)$ are polynomials, the sensitivity functions can be expressed as:

$$
\begin{aligned}
S_{y y} & =\frac{A S}{A S+B R} \\
S_{y b} & =\frac{-B R}{A S+B R} \\
S_{y u} & =\frac{B S}{A S+B R}
\end{aligned}
$$

From these expressions, it can be noted that the three sensitivity functions have the same denominator $D=A S+B R$ which determines the closed-loop poles. It can be noted that sensitivity functions are independent of $T(z)$.

The knowledge of acceptable disturbances leads to design the RST controller in terms of pole and zero assignments. Some fixed parts can be specified for the polynomials $S(z)$ 


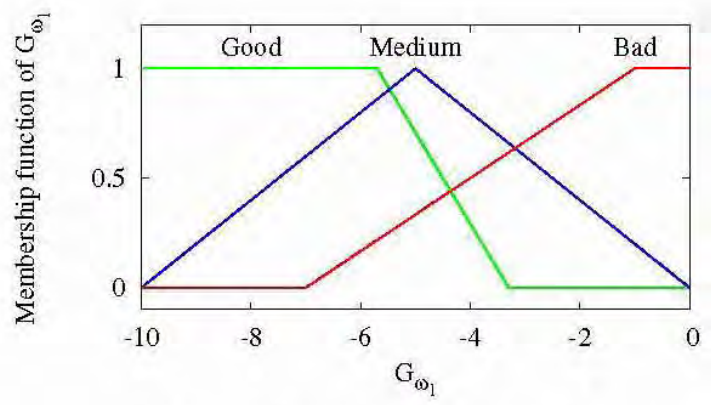

Fig. 7. Input membership function for $G_{\omega_{1}}$

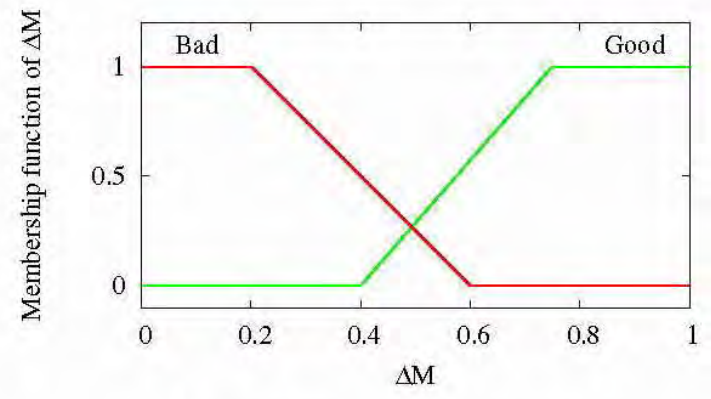

Fig. 8. Input membership function for $\Delta \mathrm{M}$

and $R(z)$. For example, to insure the output accuracy, a pole for $\mathrm{z}=1$ in $\mathrm{S}(\mathrm{z})$ is necessary for static error elimination. The closed-loop poles are chosen either for filtering effects in certain frequency regions or for improving the robustness of the closed-loop system. For an output disturbance at pulsation $\omega_{1}$, the lower the gain of $S_{y y}$ the better the attenuation of the output disturbance rejection. However it can be shown that the larger the attenuation of $S_{y y}$ at $\omega_{1}$, the larger the area of $S_{y y}$ over the zero value [15]. It can induce a increase in the maximum value of $S_{y y}$. As the maximum value of $S_{y y}$ is inversely proportional to $\Delta \mathrm{M}$, a larger output noise rejection leads to a worse robustness.

How to determine a controller which offers a trade-off between the robustness and a good rejection of disturbances? This can be described as an optimization problem by defining a cost function which qualifies the controller robustness and noise rejection properties.

Fuzzy logic is suitable to qualify the robustness of a controller and noise rejection properties [18]. Indeed the frontier between a good controller and a bad controller is not strict. For example, if one considers the modulus margin is correct if it is higher than 0.5 , it is clear that a controller will not be qualified with a bad robustness getting to good by crossing this value. With membership functions of fuzzy logic, the controller quality can be evaluated continually from bad to good across medium.

An example of membership functions qualifying the gain at $\omega_{1}\left(G_{\omega_{1}}\right)$ and the modulus margin $(\Delta \mathrm{M})$ is shown in Fig. 7 and Fig. 8. $G_{\omega_{1}}$ is normalized over $\left\{\begin{array}{lll}-10 & 0\end{array}\right\}$ and $\Delta \mathrm{M}$ uses its natural scale. The output membership function is given

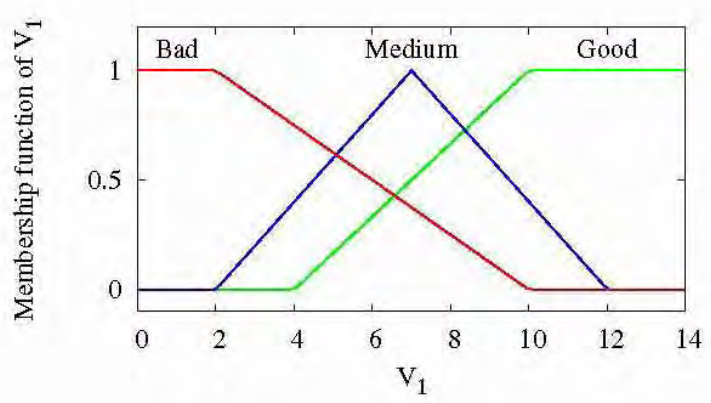

Fig. 9. Output membership functions

TABLE I

FuzZY Rules USED

\begin{tabular}{|c|c|c|c|}
\hline $\begin{array}{ll}{ }_{\Delta M} & G_{\omega_{1}} \\
\end{array}$ & Bad & Medium & Good \\
\hline Bad & $\overline{\mathrm{Bad}}$ & $\overline{\mathrm{Bad}}$ & Medium \\
\hline Good & $\mathrm{Bad}$ & Medium & Good \\
\hline
\end{tabular}

in Fig. 9. The stability robustness can be expressed by fuzzy rules defined in Tab. I. After "disfuzzyfication", the robustness analysis is quantified by the function $V_{1}=\mathrm{f}\left(G_{\omega_{1}}, \Delta \mathrm{M}\right)$ which corresponds to a surface as presented in Fig. 10. It can be seen that for $\Delta \mathrm{M}>0.5$, the lower the values of $G_{\omega_{1}}$ the better the output function $V_{1}$.

In the same way, other membership functions and fuzzy rules in relation with delay margin and other sensitivity functions can be defined to quantify constraints of robustness and satisfy the requirements of disturbance rejection performances. A weighted sum of all fuzzy functions defines the quality function to maximize.

The optimization problem is addressed by a genetic algo-

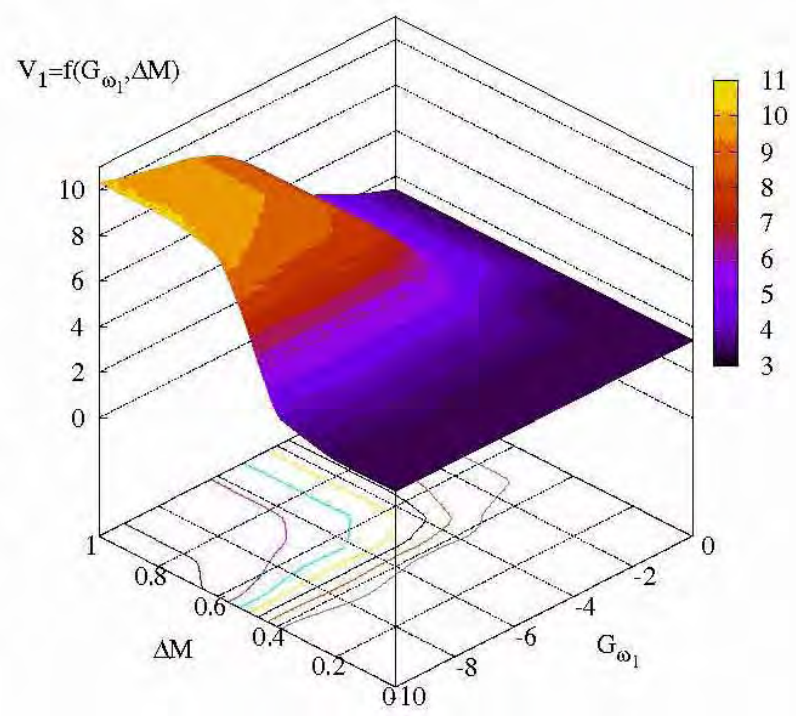

Fig. 10. Fuzzy function $V_{1}$ 


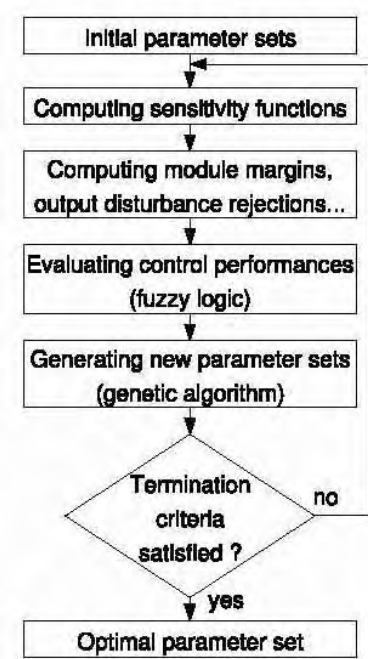

Fig. 11. Off line approach used to determine controller parameters

rithm which is a stochastic tool based on the mechanism of natural selection. Each variables $X_{i}$ (chromosomes) are coded on $n_{i}$ bits between a minimum value $\min _{i}$ and a maximum one $\max _{i}$. An initial population of strings (individual) is arbitrarily created. Genetic algorithm makes it change by using three main operations: reproduction, crossover and mutation in order to maximise an objective function (fitness value) [19]. The performed computation time can be important but it is offline, so that is not a limitation for real-time implementation.

The offline approach used to determine controller parameters is summarized in Fig. 11. This optimization problem is solved in the Matlab/Similink environment.

For SMPS application, the denominator of the sensitivity function $S_{y y}$ is chosen as follows to determine a RST controller which presents a good robustness and a good attenuation of the influence of the output disturbance at $\omega_{1}=11 \mathrm{kHz}$.

$$
D(z)=\left(1-c_{0} z^{-1}\right)\left(1+c_{1} z^{-1}+c_{2} z^{-2}\right)
$$

where $c_{0}$ is between 0.1 to $0.99,\left(1+c_{1} z^{-1}+c_{2} z^{-2}\right)$ corresponds to a pair of complex zeros for which the frequency band and the damping ratio are between $\omega_{0}=1 \cdot 10^{5} \mathrm{rad} / \mathrm{s}$ to $3 \cdot 10^{5} \mathrm{rad} / \mathrm{s}$ and $\xi_{0}=0.3$ to $1 c_{0}, \omega_{0}$ and $\xi_{0}$ constitute three degrees of freedom (or chromosomes) for the optimization problem. For each combination of $c_{0}, \omega_{0}$ and $\xi_{0}$ (i.e. each individual for the genetic algorithm), the fuzzy logic quantifiers the quality function (or fitness value). The genetic algorithm then finds the optimal solution for $c_{0}, \omega_{0}$ and $\xi_{0}$ over twenty generations with about thirty individuals for $n_{i}=16$, crossover probability $=0.9$ and mutation probability $=0.02$.

The individuals which have been tested by the genetic algorithm are plotted in Fig. 12. The corresponding optimal polynomial $R(z), S(z)$ and $T(z)$ are calculated by solving the Bezout equation [15].

$$
\begin{array}{cc}
R(z)= & 16.03 z^{2}-28.21 z+12.82 \\
S(z)= & z^{2}-0.82 z-0.18
\end{array}
$$

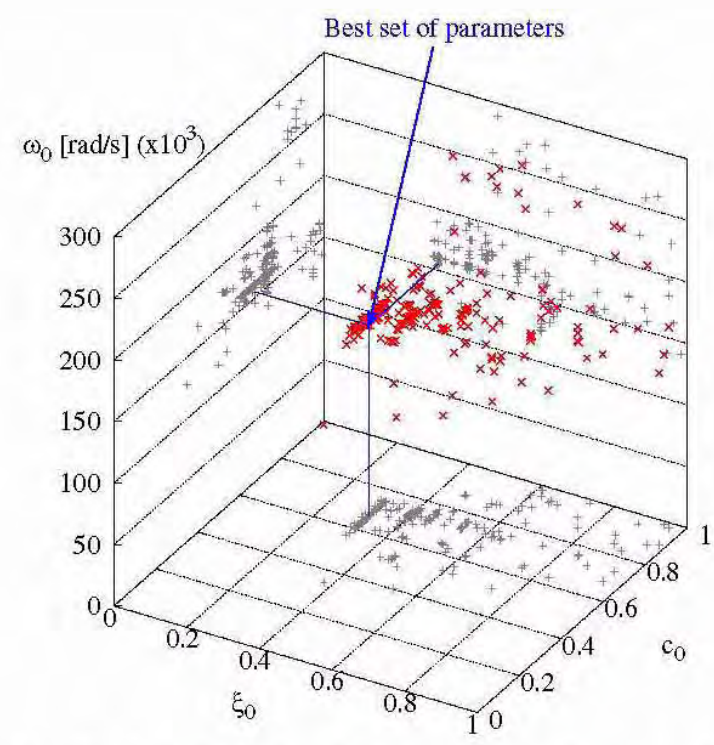

Fig. 12. Individuals which have been tested by the genetic algorithm

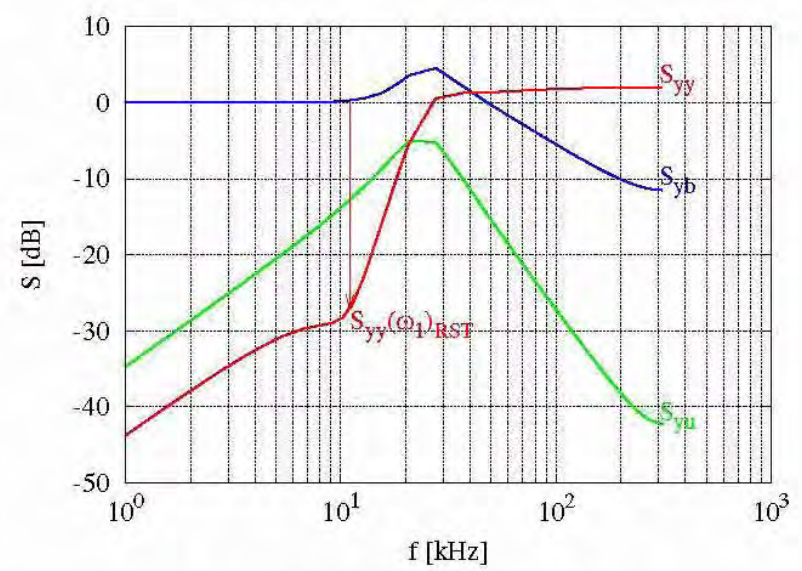

Fig. 13. Sensitivity functions for RST controller

$$
T(z)=35.11 z^{3}-86.84 z^{2}+72.62 z-20.24
$$

Since, the obtained controller is described by (14), the hardware implementation is quite simple: it just need a few memories, multipliers and adders.

The corresponding sensitivity functions and Nyquist plot of $L_{y y}$ are respectively given in Fig. 13 and 14 .

The gain of $S_{y y}$ at $\omega_{1}$ (LC filter resonance) is $-27 \mathrm{~dB}$ against $-8 \mathrm{~dB}$ for the PID controller. The good robustness is keep with $\Delta \mathrm{M}_{R S T}=0.79, \Delta \Phi_{R S T}=51^{\circ}$ and $\Delta \tau_{R S T}=2.16 \mathrm{~T}_{e}$.

\section{Simulation Results}

The simulations are performed using Simulink (Fig. 15). The buck SMPS is modeled by a hybrid model [4]. The PID controller and RST controller are computed with fixed-point algorithm. The analog-to-digital converter model has a $10-$ bit resolution and takes into account quantization, delay and saturation effects. The DPWM model has a 8-bit resolution. 


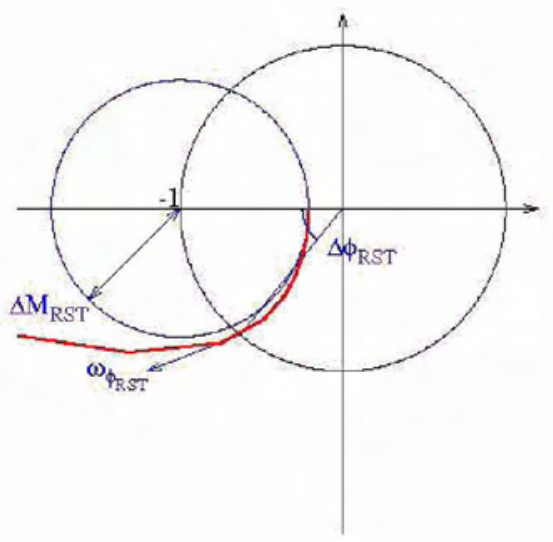

Fig. 14. Nyquist plot of $L_{y y}$ for RST controlled system

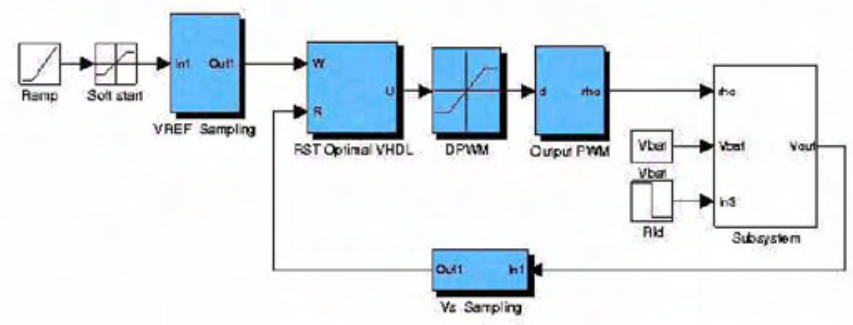

Fig. 15. Simulink model of the RST digital controller for the buck converter

The output voltages are compared during a step change of load from $10 \Omega$ to $3 \Omega$ in Fig. 16. The load transient response of RST controller is superior to PID controller since it results in shorter rise time and produces smaller undershoot.

\section{CONCLUSION}

This paper details an off-line automated design of a RST digital controller. Robust RST controller offers better performances compared to a classical digital PID controller. As the design approach is based on robustness, neither parameters uncertainty (due to aging or manufacturing disparity) nor neglected physical phenomena will lead to an unstable system. An real time auto tuning or parameter estimator is not necessary, so RST implementation can be simply achieved using

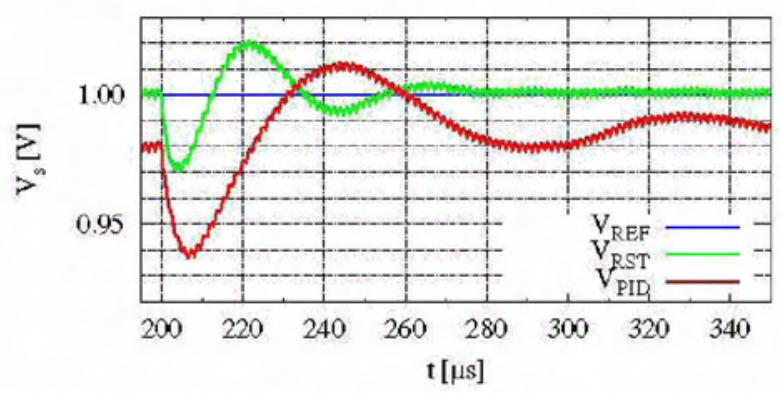

Fig. 16. Comparison of output voltage responses to load perturbation for the RST and PD controller one A-to-D converter and standard CMOS logic. Experimental study are currently performed using an Actel ProAsic3 FPGA board plus an additional analog $\mathrm{I} / \mathrm{O}$ board. It will be possible to estimate the impact of fixed point computing and monolithic integration from experimental results as FPGA design can be translated at no cost into an ASIC. PID like RST controller requires a fast A-to-D converter. It will probably be the bigger energy consumer in the digital control. Energy consumption is one of the most important criteria when considering digital controllers instead of analog controllers in embedded devices. So this point will be considered in future works.

\section{REFERENCES}

[1] V. Kursun, S. Narendra, V. K. De, and E. Friedman, "High input voltage step-down dc/dc converters for integration in a low voltage cmos process," Proc. of IEEE ISCAS, 2004.

[2] H. Deng, X. Duan, Y. Ma, A. Huang, and D. Chen, "Monolithically integrated boost converter based on 0.5 um cmos process," IEEE Transactions on Power electronics, vol. 20 , no. 3, pp. 628-638, 2005.

[3] V. Pinon, B. Allard, and C. Garnier, "High-frequency monolithic dcidc converter for system-on-chip power management high-frequency monolithic $\mathrm{dc} / \mathrm{dc}$ converter for system-on-chip power management," Proceedings of IEEE ISP SD, Naples, June, 2006.

[4] S. Trochut, B. Allard, X. Lin-Shi, and J. M. Rétif, "Control design for integrated switch-mode power supplies: a new challenge ?' Proc. of the IEEE Power Electronics Specialist's Conference, 2004, cdrom.

[5] T. Martin and S. Ang, "Digital control for switching converters," Proc of IEEE ISIE, vol. 2, pp. 480-484, 1995.

[6] H. Matsuo, Y. Mimura, Y. Nakao, F. Kurokawa, and M. Sasaki, "Novel digital controller for the pwom and/or pfm controlled dwitching dc/dc converters," Proc of IEEE INTELEC, vol. 2, pp. 65-68, 2001.

[7] J. L. G. Hung and R. Nelms, "Pid controller modifications to improve steady-state performance of digital controllers for buck and boost converters," Proc of IEEE APEC, vol. 1, pp. 381-388, 2002.

[8] D. Maksimovic, R. Zane, and R. Erickson, "Impact of digital control in power electronics," Proc of IEEE ISPSD, pp. 13-22, 2004.

[9] S. Musunuri, P. Chapman, J. Zou, and C. Liu, "Design issues for monolithic dc-dc converters," IEEE Transactions on Power electronics, vol. 20 , no. 3 , pp. $639-649,2005$.

[10] G. Wei and M. Horowitz, "A fully digital, energy-efficient, adaptative power-supply regulator, IEEE Joumal of Solid-State Circuits, vol. 34, no. 4, pp. 520-528, 1999.

[11] B. J. Patella, A. Prodic, A. Zirger, and D. Maksimovic, "High-frequency digital pwm controller ic for dc-dc converters," IEEE Transactions on Power electronics, vol. 18, no. 1, pp. 438-446, 2003.

[12] W. Stefanutti, P. Mattavelli, S. Saggini, and M. Ghioni, "Autotuning of Digitally Controlled Buck Converters Based on Relay Feedback," in Power Electronics Specialists Conference, 2005. PESC '05. IEEE 36th, Recife, 2005, pp. 2140-2145.

[13] Z. Zhao, H. Li, A. Feizmohammadi, and A. Prodic, "Limit-cycle Based Auto-tuning System for Digitally Controlled Low-power SMPS," in Appited Power Electronics Conference and Exposition, 2006. APEC'06. Twenty-First Annual IEEE, Mar. 19-23, 2006, pp. 1143-1147.

[14] W. Xiao and W. G. Dunford, "Fuzzy logic auto-tuming applied on DCDC converter," in Industrial Electronics Society 2004. IECON 2004. 30th Annual Conference of IEEE, vol. 3, Nov, 2-6, 2004, pp. 26612666.

[15] I. Landau, Identification et commande des systèmes. Hermes, 1993, 2nd edition.

[16] I. LANDAU and A. KARIMI, "Robust digital control using pole placement with sensitivity function shaping method," Int. J. Robust Nonlinear Control, no. 8, pp. 191-210, 1998.

[17] R. W. Erickson and D. Maksimovic, Fundamentals of Power Electronics. Kluwer, 2001, 2nd edition.

[18] C.C.Lee, "Fuzzy logic in control systems: Fuzzy logic controller-part i," IEEE Trans. On system, Man and Cybernetics, vol. 20, no. 2, pp. 404-408, 1990.

[19] D.Beasley and al., "An overview of genetic algorithms: Part 1, fundamentals," University Computing, vol. 15, no. 2, pp. 58-69, 1993. 